\title{
EVALUATION OF SERUM OXIDATIVE STRESS, VITAMIN B12 AND HOMOCYSTEINE LEVELS IN PRE-ECLAMPTIC PREGNANT WOMEN
}

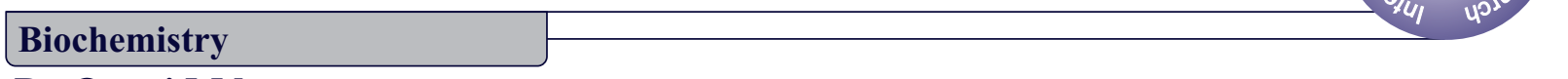

Dr Quazi Md Junior Resident, Dept. of Biochemistry, IPGME\&R, 244 A.J.C. Bose Road, KolkataTajuddin*

\section{Dr Mousumi} Mukhopadhyay Dr Sambhu Nath Bandyopadhyay
700020, West Bengal, India. * Corresponding Author

Professor \& HOD, Dept. of Biochemistry, IPGME\&R, 244 A.J.C. Bose Road, Kolkata700020, West Bengal, India.

Associate Professor, Dept. of Gynecology and Obstetrics, IPGME\&R, 244 A.J.C. Bose Road, Kolkata-700020, West Bengal, India.

\section{ABSTRACT}

Background: Pre-eclampsia is a new onset pregnancy induced hypertensive disorder associated with proteinuria. Deleterious effect of hyperhomocysteinemia and subsequent oxidative stress may be one of the etiologies. Level of vitamin B12 may be of concern as it is metabolically closely related to homocysteine. Aim: The aim of the study is to evaluate the levels of serum oxidative stress, vitamin B12 and homocysteine in preeclamptic women. Materials and methods: This cross sectional observational study was conducted in Institute of Post Graduate Medical Education \& Research, Kolkata. Plasma levels of homocysteine, vitamin B12 and Malondialdehyde (MDA) as a marker of oxidative stress were estimated in diagnosed 40 pre-eclamptic cases as well as in equal number of age matched pregnant control (group I) and non-pregnant control (group II). MDA was measured by Kei-Satoh's method. Homocysteine and vitamin B12 were estimated by chemiluminescence. Results: A moderate increase of MDA observed in normal pregnancy where as this increase is marked in pre-eclampsia. On the other hand vitamin B12 as well as homocysteine level decline during normal pregnancy but in pre-eclampsia significant hyper-homocysteinemia seen in contrast to low vitamin B12 level. Conclusions: Increased oxidative stress, hyperhomocysteinemia and low vitamin B12 level may play a pivotal role in pathophysiology of pre-eclampsia. Clinical significance: As clinical alarm of pre-eclampsia is always very late, so estimation of these biochemical parameters as a screening test for early prediction can be of clinically valuable.

\section{KEYWORDS}

\section{Pre-eclampsia, Malondialdehyde (MDA), Homocysteine, Vitamin B12}

\section{INTRODUCTION:}

Pre-eclampsia is a systemic vascular hypertensive disorder occurring in $2-8 \%$ of pregnant women. ${ }^{[1]}$ It is the second leading cause of direct maternal and fetal death. ${ }^{[2]}$ Pre-eclampsia is defined classically as newonset pregnancy induced hypertensive disorder associated with proteinuria or with other organ dysfunction in absence of proteinuria after 20 weeks of gestation.

Despite considerable research etiology of eclampsia is poorly elucidated. There should have some placenta related factors responsible behind pre-eclampsia, as delivery of placenta terminates the features of pre-eclampsia. Among the multifactorial etiology, placental hypoperfusion and endothelial dysfunction are considered to be triggering factor for increase level of placenta related circulatory parameters.

It has been suggested that free radical induced endothelial cell injury might be one of the etiologies. ${ }^{[3]}$ An imbalance between oxidant and antioxidant status has also been reported in pre-eclamptic, but the results are conflicting. Beside this, hyper-homocysteinemia might damage the vascular endothelium of the developing placenta by promoting oxidative stress, thereby increasing contractile response and the production of pro-coagulants and vasoconstrictor. ${ }^{[4]}$ Plasma homocysteine is normally lower throughout pregnancy than in the non-pregnant state and also closely dependent on vitamin B12 intake. ${ }^{[5]}$ Thus peri conceptional nutritional status of vitamin B12 may affects homocysteine level and influence pathophysiology of pre-eclampsia.

In this study we measured the extent of oxidative stress by measuring Malondialdehyde (MDA) as well as homocysteine and vitamin B12 level in preeclamptic women and compared them with normotensive pregnant and non-pregnant control to find out if there is any significant correlation present.

\section{MATERIALS AND METHODS:}

The study was conducted in the Dept. of Biochemistry and the Dept. of Obstetrics \& Gynaecology, IPGME\&R, Kolkata during January, 2018-June 2019. Altogether, 40 diagnosed preeclamptic women having new onset Systolic Blood Pressure (SBP) $>140 \mathrm{~mm} \mathrm{Hg}$ and/ or Diastolic Blood Pressure (DBP) $>90 \mathrm{~mm} \mathrm{Hg}$, with proteinuria $\geq 300 \mathrm{mg} / 24 \mathrm{hrs}$ or protein creatinine ratio $\geq 0.3$ appearing after 20 weeks of gestation, were taken as cases. Whereas 40 gestational agematched normotensive and non proteinuric pregnant women were taken as control group I and 40 age-matched apparently healthy non pregnant women were taken as control group II. Pregnant women with other co-morbidity and on vitamin B12 or antifolate drugs were excluded from the study.

$5 \mathrm{ml}$ of clotted venous blood and spot urine were taken as sample and relevant information and clinical findings were recorded in predesigned data sheet.

Malondialdehyde (MDA), a Thiobarbituric acid reactive substances (TBARS), was measured by Kei-Satoh's method as described by Kei Satoh (1978) in his research study, by formation of Thiobarbituric acid (TBA)-MDA adduct and production of pink colour which is measured spectrophotometrically at $532 \mathrm{~nm} .{ }^{[6]}$ Serum homocysteine and vitamin B12 were estimated by chemiluminescence assay (CLIA) and spot urinary protein was measured by Pyrogallol red method.

\section{RESULTS:}

Collected data was expressed as mean \pm standard deviation (Table 1) $\&$ comparison done after student's unpaired t-test .Statistical analysis was done by SPSS-24.0. P value $<0.05$ was considered significant.

Table 1: Clinical and Biochemical data of case and control groups

\begin{tabular}{|c|c|c|c|}
\hline Variable & Cases & $\begin{array}{c}\text { Control group } \\
\text { I (Pregnant) }\end{array}$ & $\begin{array}{c}\text { Control group } \\
\text { II } \\
\text { (Non pregnant) }\end{array}$ \\
\hline $\mathrm{n}$ & 40 & 40 & 40 \\
\hline Age (years) & $26.83 \pm 3.23$ & $25.78 \pm 3.62$ & $26.38 \pm 4.55$ \\
\hline SBP (mm of Hg) & $150.10 \pm 5.78$ & $118.55 \pm 9.28$ & $118.70 \pm 8.84$ \\
\hline DBP (mm of Hg) & $95.40 \pm 3.77$ & $75.45 \pm 5.36$ & $77.05 \pm 5.44$ \\
\hline $\begin{array}{c}\text { Protein creatinine } \\
\text { ratio }\end{array}$ & $0.64 \pm 0.26$ & $0.25 \pm 0.05$ & $0.24 \pm 0.09$ \\
\hline MDA (nmol/ml) & $2.84 \pm 0.30$ & $1.89 \pm 0.16$ & $0.93 \pm 0.20$ \\
\hline Vitamin B12 $(\mathrm{pg} / \mathrm{ml})$ & $179.1 \pm 31.77$ & $232.5 \pm 70.01$ & $271.08 \pm 47.60$ \\
\hline $\begin{array}{c}\text { Homocysteine } \\
(\mu \mathrm{mol} / \mathrm{lt})\end{array}$ & $20.21 \pm 2.78$ & $8.41 \pm 3.10$ & $18.68 \pm 2.92$ \\
\hline
\end{tabular}

The serum MDA level was moderately increased $(\mathrm{p}<0.05)$ in pregnant control or group I $(1.89 \pm 0.16 \mathrm{nmol} / \mathrm{ml})$ than non-pregnant control or group II $(0.93 \pm 0.20 \mathrm{nmol} / \mathrm{ml})$. In preeclamptic women the rise in MDA levels $(2.84 \pm .30 \mathrm{nmol} / \mathrm{ml})$ was more marked and significant $(\mathrm{p}<0.05)$ than two control groups. (Figure 1) 


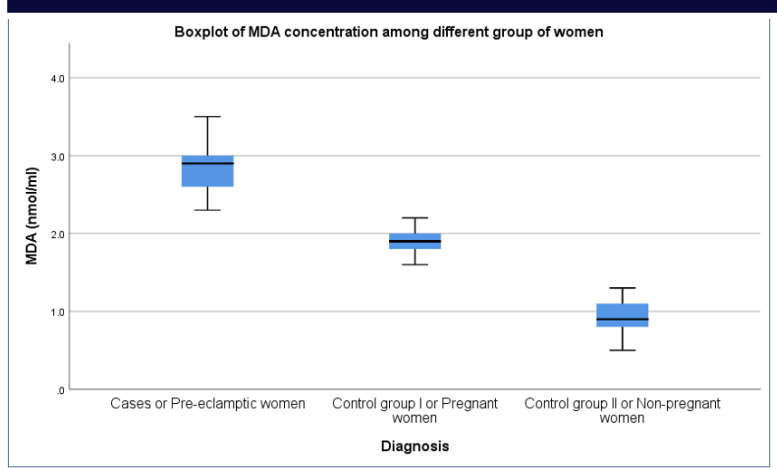

Figure 1: Distribution of MDA concentration in case and control groups

It has been observed that significant decrease $(p<0.05)$ of serum vitamin B12 level in preeclampsia $(179.1 \pm 31.77 \mathrm{pg} / \mathrm{ml})$ than both control groups. On the other hand level of vitamin B12 in control group I $(232.5 \pm 70.01 \mathrm{pg} / \mathrm{ml})$ is also significantly reduced $(\mathrm{p}<0.05)$ than control group II (271.08 $\pm 47.60 \mathrm{pg} / \mathrm{ml})$.(Figure 2)

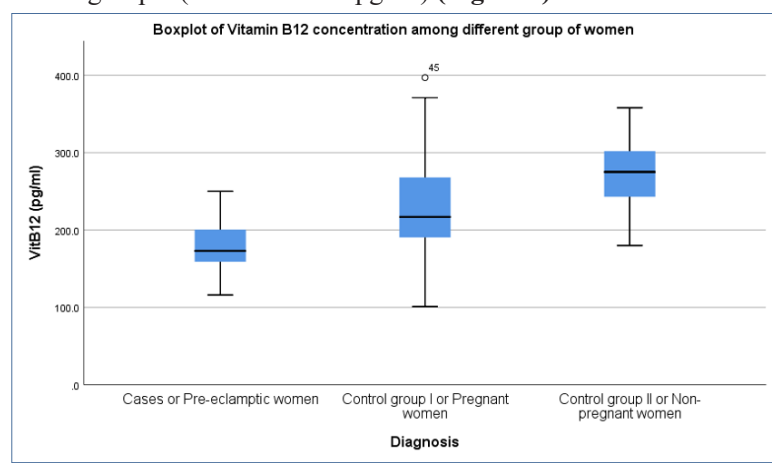

Figure 2: Distribution of Vitamin B12 concentration in case and control groups

Significant statistical difference $(\mathrm{p}<0.05)$ has been found in homocysteine level between cases $(20.21 \pm 2.77 \mu \mathrm{mol} / \mathrm{lt})$ and control group I $(8.41 \pm 3.10 \mu \mathrm{mol} / \mathrm{lt})$ and control group II $(18.68 \pm 2.92$ $\mu \mathrm{mol} / \mathrm{lt})$. The difference in homocysteine level in between control group I and control group II was also statistically significant $(\mathrm{p}<0.05)$. (Figure 3)

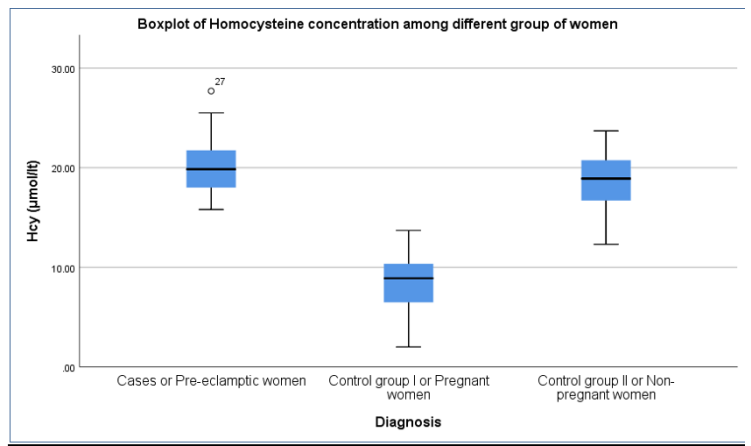

Figure 3: Distribution of Homocysteine concentration in case and control groups

\section{DISCUSSION:}

Pre-eclampsia, a multisystem pregnancy specific hypertensive disorder is one of the leading causes of maternal and fetal death. Despite various researches pathophysiology of pre-eclampsia is poorly explored. Recent study shows that defective trophoblastic invasion with associated uteroplacental hypoperfusion are the initiators which lead to an increase in oxidative stress and a state of relative ischemia during intermittent perfusion. A pivotal role of enhanced placental superoxide generation leading to oxidative stress is increasingly recognized ${ }^{[7]}$. Furthermore, homocysteine contributes to oxidative stress not only through the increased ROS generation but also involves the suppression of antioxidant capability of endothelial cells ${ }^{[8]}$. In examining the relationship of serum vitamin B12 levels to homocysteine levels, previous study highlight the utility of homocysteine in the clinical investigation of vitamin B12 deficiency ${ }^{[9]}$. Therefore our study was undertaken to estimate the level of oxidative stress by measuring a lipid peroxidation product MDA and also the levels of homocysteine and vitamin B12, to further explore the association. 40 diagnosed pre-eclamptic women were selected as cases along with an equal number of age matched apparently healthy pregnant and non-pregnant women selected as controls based on inclusion and exclusion criteria.

In this study it was found that mean-MDA level was significantly higher in pre-eclamptic women compared to the control group I and control group II. This is corroborated with the findings that one of the first biomarkers of lipid peroxidation found to be elevated in preeclampsia was malondialdehyde ${ }^{[10]}$. In accordance with our study, it is proposed that preeclampsia develops when the systemic inflammatory process, common to all women in the second half of their pregnancies, causes one or other maternal system to decompensate ${ }^{[1]}$.

It is also found that mean vitamin B12 level in pre-eclamptic women was significantly lower than both control groups. Our results are in agreement with previous research which showed total serum B12 level decline during pregnancy due to hemodilution and increased foetal demand. ${ }^{[12]}$ Recent observational studies also suggest that maternal vitamin B-12 deficiency is associated with increased risk of pregnancy complications, such as preeclampsia ${ }^{[13]}$.

Hyperhomocysteinemia is a risk factor for endothelial dysfunction and vascular disease just as is pre-eclampsia. It may lead eventually to preeclampsia with subsequent activation of various factors. In the present study the mean homocysteine level in pre-eclamptic women found to be significantly higher than both control groups. The lower level of homocysteine in pregnant controls may be observed due to physiological response to pregnancy, increase in estrogen level, hemodilution or increased demand for methionine by both mother and fetus ${ }^{[14]}$. This decrease in homocysteine level does not occur in preeclampsia which conforms to study done by Rajkovic et al ${ }^{[15]}$.

\section{CONCLUSION:}

Therefore, it can be concluded that a moderate increase of oxidative stress seen in normal pregnancy whereas this increment is marked in pre-eclampsia which may play an important role in pathophysiology of pre-eclampsia. This study also suggests that hyperhomocysteinemia coupled with low vitamin B12 in early pregnancy may harbinger the onset of pre-eclampsia. Thus our study indicates that estimation of these biochemical parameters as a screening test for early prediction of the onset of pre-eclampsia would be clinically valuable to identify and monitor those who are prone to develop pre-eclampsia and also to avoid undesired adverse pregnancy outcome. However further longitudinal interventional studies with larger sample should be carried out to evaluate and strengthen the association.

\section{REFERENCES:}

1) Brown MA,Lindheimer MD,de Swiet M,et al. The classification and diagnosis of the hypertensive disorders of pregnancy: statement from the International Society for the Study of Hypertension in Pregnancy (ISSHP). Hypertension in Pregnancy. 2001;20: IXXIV

2) Cunningham F,Lenevo K,Bloom S, et al. Hypertension in Pregnancy. Williams Obstetrics, The McGraw Hill Companies,USA. 2010;23:706-707.

3) Shane B.Stokstad EL. Vitamin B12-folate interrelationships. Annual Review of Tutrition $1985 ; 5: 115-141$.

4) Welch GN,Loscalzo J. Homocysteine and atherosclerosis. N Engl J Med.1998;338; 1042-1050.

5) Vollset SE,Refsum H,Irgens LM,et al. Plasma Total Homocysteine, Pregnancy Complications, and Adverse Pregnancy Outcomes: the Hordaland Homocysteine Study. Am J Clin Nutr. 2000;71:962-968

6) Kei Satoh. Serum lipid peroxide in cerebrovascular disorders determined by a new colorimetric method. Clin Chem Acta. 1978;90:37-43.

7) Hubel CA. Oxidative stress in the pathogenesis of preeclampsia. Proc Soc Exp Bio Med. 1999;222:222-235.

8) Frey D,Braun O,Briand C,et al.: Structure of the mammalian NOS regulato dimethylarginine dimethylaminohydrolase: a basis for the design of specific inhibitors. Structure 2006;14:901-911.

9) Robertson J,Iemolo F,Stabler SP,et al. Vitamin B12, homocysteine and carotid plaque in the era of folic acid fortification of enriched cereal grain products. CMAJ 2005;172(12):1569-73

10) Hubel CA,Roberts JM,Taylor RN,et al. Lipid peroxidation in pregnancy: new perspectives on preeclampsia. Am J Obstet Gynecol. 1989;161:1025-1034

11) Redman CWG,Sargent IL.Placental Stress and Pree-clampsia: A Revised View. Placenta 30, Supplement A, Trophoblast Research.2009;23:S38-S42.

12) Hure AJ,Collins CE,Smith R. A longitudinal study of maternal folate and vitamin B12 status in pregnancy and postpartum, with the same infant markers at 6 months of age. Matern Child Health J 2012;16:792-801.

13) Refsum H,Yajnik CS,Gadkari M,et al. Hyperhomocysteinemia and elevated methylmalonic acid indicate a high prevalence of cobalamin deficiency in Asian Indians. Am J Clin Nutr 2001;74:233-41. 
14) Andersson A,Hultberg B,Brattstrom L,Isaksson A. Decreased serum homocysteine in pregnancy. Eur J Chem Clin Biochem. 1992;30:377-379.

15) Rajkovic A,Catalano PM,Malinow MR. Elevated Homocysteine Levels with Preeclampsia. Obstet Gynaecol 1997;90:168-171. 